\title{
Travail collectif pluridisciplinaire dans la prévention des risques professionnels complexes : ressources et contraintes du contexte au Québec et en France \\ Collective Multidisciplinary Work in the Prevention of Complex Occupational Risks: Resources and Constraints in Quebec and France \\ Trabajo colectivo multidisciplinario en la prevención de riesgos ocupacionales complejos: recursos y limitaciones en Quebec y Francia
}

\author{
Marie Bellemare, Sandrine Caroly et Daniel Prud'homme
}

Volume 74, numéro 2, printemps 2019

URI : https://id.erudit.org/iderudit/1062083ar

DOI : https://doi.org/10.7202/1062083ar

Aller au sommaire du numéro

Éditeur(s)

Département des relations industrielles de l’Université Laval

ISSN

0034-379X (imprimé)

1703-8138 (numérique)

Découvrir la revue

Citer cet article

Bellemare, M., Caroly, S. \& Prud'homme, D. (2019). Travail collectif pluridisciplinaire dans la prévention des risques professionnels complexes : ressources et contraintes du contexte au Québec et en France. Relations industrielles / Industrial Relations, 74(2), 242-265.

https://doi.org/10.7202/1062083ar

\section{Résumé de l'article}

Dans le cadre d'une étude menée au sein de services de santé au travail au Québec et en France, nous nous intéressons aux conditions favorables aux pratiques de travail collectif pluridisciplinaire (TCP). Face à des problèmes de santé complexes, tels que les troubles musculosquelettiques (TMS) ou les troubles de santé psychologique (TPS), des professionnels de différents métiers sont amenés à collaborer, notamment pour mener des actions de prévention primaire ayant pour cible les conditions de réalisation du travail. Le contexte dans lequel oeuvrent, d'une part, les équipes de santé au travail (ESAT) au Québec et, d'autre part, les services de santé au travail interentreprises (SSTi) en France diffèrent en raison de leur histoire, leurs cadres juridique et institutionnel, leurs missions et dispositifs.

En étudiant les actions en milieu de travail menées par les acteurs de ces services, à partir de données recueillies par le biais d'observations et d'entretiens réalisés dans les deux contextes, nous examinons les formes de travail collectif pluridisciplinaire en prévention des TMS. Ceci nous a permis de mettre en lumière les contraintes et les ressources qu'offre, à cet égard, chacun des contextes. Nous constatons que le contexte québécois, avec son approche de santé publique qui prescrit une démarche structurée faisant appel à la pluridisciplinarité, permet des formes plus élaborées de travail collectif grâce au partage d'outils communs et aux prises de décisions collectives quant aux modalités de l'intervention. Dans le contexte français, bien que les formes du travail collectif pluridisciplinaire soient moins développées, le mandat de prévention assuré par les services de santé au travail permet un élargissement du périmètre d'action que l'on ne retrouve pas dans le contexte québécois. Ces résultats interrogent les marges de manoeuvre offertes par les dispositifs en vue d'une prévention durable et efficace des risques professionnels.
Tous droits réservés (C Département des relations industrielles de l’Université Laval, 2019
Ce document est protégé par la loi sur le droit d'auteur. L’utilisation des services d'Érudit (y compris la reproduction) est assujettie à sa politique d'utilisation que vous pouvez consulter en ligne. 


\title{
Travail collectif pluridisciplinaire dans la prévention des risques professionnels complexes: ressources et contraintes du contexte au Québec et en France
}

\author{
Marie Bellemare, Sandrine Caroly et Daniel Prud'homme
}

La complexification des problématiques de santé au travail observée depuis les dernières décennies nous amène à nous intéresser aux services de santé au travail et à la manière dont les interventions de prévention sont menées en entreprise. Les troubles musculosquelettiques, bien présents dans les milieux de travail, constituent un exemple de problème de santé complexe. En comparant deux contextes différents, ceux de la France et du Québec, nous avons examiné en quoi chacun d'eux comportait des ressources et des contraintes pour le travail collectif pluridisciplinaire dans le cadre des interventions de prévention effectuées par les services de santé au travail. Ces résultats nous mènent à réfléchir aux évolutions souhaitables dans chacun de ces deux contextes.

MOTS-CLÉs: intervention, troubles musculosquelettiques, médecine du travail, santé au travail, Québec, France.

\section{Introduction}

Les services de santé au travail constituent un rouage important de la prévention des risques professionnels. Or, le contexte de ces services varie d'un État à l'autre et leur avenir est objet de débats. Dans une recherche portant sur le rôle des médecins du travail, nous avons comparé la situation de la France et du Québec (Caroly et al., 2011), ce qui nous a amenés à souligner la composi-

Marie Bellemare, professeure associée, Département des relations industrielles, Université Laval, chercheuse régulière au CINBIOSE (marie.bellemare.1@ulaval.ca).

Sandrine Caroly, professeure des universités, Université Grenoble-Alpes, chercheuse au PACTE, France (sandrine.caroly@univ-grenoble-alpes.fr).

Daniel Prud'homme, chargé d'enseignement et candidat au doctorat, Département des relations industrielles, Université Laval (Daniel.Prudhomme@rlt.ulaval.ca). 
tion pluridisciplinaire des équipes de santé au travail au Québec. Cette situation prévaut en territoire québécois depuis la réforme du régime de santé au travail de 1979 alors que, en France, la pluridisciplinarité a été imposée depuis 2004. Celle-ci devient un enjeu important pour répondre à l'évolution des problématiques santé-travail, notamment à cause de leur complexification. Examiner comment les acteurs des services de santé au travail sont en mesure de collaborer entre eux apparait essentiel, surtout si l'on souhaite parfaire le travail collectif entre les différents métiers de la prévention. L'objectif de cet article est de comprendre dans quelle mesure chacun des contextes, québécois et français, offre des ressources pour mener le travail collectif pluridisciplinaire (TCP).

Après avoir exposé le contexte des pratiques pluridisciplinaires au Québec et en France dans la section problématique, nous présentons le cadre conceptuel et les questions qui ont guidé nos analyses. La méthodologie de la recherche est ensuite expliquée, puis nous exposons les modalités du TCP dans chacun des contextes, pour ensuite discuter des ressources et contraintes associées à ces deux différents contextes.

\section{Problématique}

La prévention des risques professionnels s'articule sur trois niveaux et les actions de prévention qui nous intéressent ici relèvent de la prévention primaire (OMS, 1999): elles consistent à intervenir de manière à changer les conditions de réalisation du travail qui peuvent porter atteinte à la santé. C'est justement à ce type d'intervention que s'intéresse notre recherche en étudiant plus particulièrement les interventions externes visant la prévention des troubles musculosquelettiques (TMS) dans les entreprises.

Les recherches sur l'efficacité des interventions en santé au travail tendent à montrer l'importance du contexte de l'intervention (Berthelette, 2002). Les milieux de travail présentent en effet des caractéristiques plus ou moins favorables aux interventions de prévention (Baril-Gingras, Bellemare et Brun, 2006; Baril-Gingras, Bellemare et Brisson, 2012), selon leur taille, leur situation économique, etc. Au-delà de l'établissement lui-même, et comme le suggère le modèle de production des changements en milieu de travail de Baril-Gingras, Bellemare et Brun (2004), un contexte plus large influence les pratiques des intervenants. Ils convient donc de s'intéresser au cadre juridique et institutionnel dans lequel prennent place les interventions des équipes de santé au travail (ESAT) au Québec et celles des services de santé au travail interentreprises (SSTi) en France. Nous caractérisons d'abord le contexte québécois, puis celui de la France, pour ensuite faire ressortir les ressemblances et divergences entre eux. 
Au Québec: une approche pluridisciplinaire de santé publique, centrée sur le milieu de travail, mais ne couvrant pas tous les salariés

Le régime québécois de prévention des risques professionnels: une approche de santé publique

Au Québec, le domaine de la santé au travail a été marqué par l'avènement de la Loi sur la santé et la sécurité au travail (LSST) mise en place en 1979. Le législateur a alors fait le choix de confier au réseau public de santé la responsabilité de fournir les services de santé au travail. L'un des objectifs de cette réforme était de mettre à la disposition des entreprises - en particulier, des petites - et des travailleurs diverses ressources spécialisées en SST (Berthelette et Pineault, 1992). II s'agissait alors d'assurer l'indépendance de ces ressources par rapport à l'employeur, tout en évitant de créer un réseau parallèle au réseau de santé publique. De plus, on séparait ces services de santé des services d'indemnisation et d'inspectorat, ceux-ci étant assumés par la Commission de la santé et de la sécurité du travail (CSST'1), organisme maître d'œuvre de la loi.

Le programme de santé spécifique à l'établissement (PSSE): un des moyens prévus par la loi pour protéger la santé des travailleurs

L'employeur a, depuis, un devoir général de prévention, et ses obligations dépassent le contenu de la règlementation sur les risques. En effet, il doit: «prendre les mesures nécessaires pour protéger la santé et assurer la sécurité et I'intégrité physique du travailleur» (LSST, article 51). La loi établit également la priorité de l'élimination du risque à la source (article 2). Elle prescrit, en outre, un ensemble de moyens visant la «prise en charge » de la santé et de la sécurité, à l'échelle des établissements, dont le programme de santé spécifique à l'établissement (PSSE). Élaboré par le médecin responsable du réseau de la santé publique, il est intégré au programme de prévention de l'entreprise. Ce PSSE comporte (LSST, article 113) des mesures de surveillance environnementale, de surveillance médicale, des activités de formation et d'information, et, enfin, de soutien à l'organisation des premiers soins (Drouin et al., 2004: 331).

\section{Les ESAT: une pluridisciplinarité préconisée dès la mise en application de la loi}

Même si la loi (LSST, article 112) ne mentionne explicitement que le médecin, appelé "médecin responsable», comme faisant partie de I'ESAT, les équipes comprennent également des infirmières et des techniciens en hygiène du travail. La pluridisciplinarité a ainsi été établie dès la mise en application de la loi. En ce qui concerne les médecins², leurs activités principales (Plante et Bhérer, 2006: 
20) consistent ainsi à: 1-visiter régulièrement les lieux de travail; 2-prendre connaissance en continu des informations nécessaires à l'accomplissement de leurs fonctions; 3-définir des programmes de santé centrés sur l'élimination des expositions aux risques professionnels; 4- déterminer les éléments de surveillance médicale ou biologique pertinents; 5 -informer le travailleur de toute situation l'exposant à un danger pour sa santé, sa sécurité ou son intégrité physique, ainsi que de toute altération à sa santé. Les infirmières et les techniciens en hygiène du travail participent à ces missions en assumant certaines fonctions reliées à leur compétence propre. Par exemple, les techniciens assument la surveillance environnementale des risques identifiés et les infirmières s'assurent de l'organisation des premiers soins et des premiers secours dans l'établissement.

\section{Une mise en application restreinte de la loi affectant} la prestation des services en santé au travail

L'une des particularités du régime québécois est que la portion de la loi encadrant l'offre de services en santé au travail ne s'applique pas à l'ensemble des entreprises. Lors de l'adoption de la LSST, les établissements ont été classés, selon leur secteur d'activités économiques, en six groupes, dont deux ont alors été déclarés prioritaires, bénéficiant ainsi des quatre mécanismes prévus à la loi, ainsi qu'un troisième groupe qui n'est couvert que partiellement, mais auquel le PSSE s'applique. Bien qu'il ait été prévu alors que les autres groupes seraient progressivement priorisés à leur tour, ceci ne s'est pas réalisé. De telle sorte que, encore en 2015 , les ESAT ne couvraient que $20 \%$ des entreprises, $25 \%$ des travailleurs et que leurs interventions se limitaient toujours aux entreprises des secteurs prioritaires I, II et III. De plus, selon Plante, Bherer et Vézina (2010), le développement de la prestation de services en santé au travail est aussi limité par le fait que la CNESST n'a pas indexé les budgets, alors que le nombre d'entreprises suivies par les ESAT s'est accru. Ceci expliquerait, en partie, les difficultés rencontrées par les équipes lors du recrutement de «médecins responsables».

\section{En France: une approche médicale centrée sur le travailleur et sur le milieu de travail, couvrant tous les salariés}

\section{À l'origine, le médecin du travail était un acteur principal de la santé au travail}

D'après les recherches de l'équipe de Rosental (Buzzi, Devinck et Rosental, 2006), la médecine du travail nait à la fin du 19 e siècle avec la montée en puissance de l'hygiène industrielle et de la reconnaissance des maladies professionnelles. Au cours de la Première Guerre mondiale, la médecine du travail se développe en milieu industriel. Elle est marquée par des visites d'embauche avec sélection et 
orientation de la main-d'œuvre. II s'agit de faire du médecin du travail un expert soucieux de la productivité et des intérêts de l'employeur, en même temps qu'un hygiéniste au service de la santé ouvrière. Dans les deux décennies suivantes apparait le terme de «médecine du travail». En 1942, la loi rend obligatoire les services médicaux pour les établissements de plus de 50 salariés. Après la Seconde Guerre mondiale, I'officialisation de la médecine du travail, qui est dorénavant régie par la Loi de 1946, repose sur le principe «d'éviter l'altération de l'état de santé des salariés du fait de leur travail », mais elle continue à s'inscrire dans une gestion de la main-d'œuvre qui a pour toile de fond d'amélioration de la productivité. Le médecin du travail fonde sa pratique sur l'aptitude médicale, avec pour objectif la sélection des salariés particulièrement adaptés à un ou plusieurs types de contraintes (Davezies, 2007). Le modèle de la santé porté ici par le médecin est celui de l'absence de maladie: le médecin du travail fait un «interrogatoire» dans son cabinet médical afin de dépister des pathologies invalidantes, telles que la silicose ou la tuberculose. Selon Davezies (1998), le certificat d'aptitude délivré alors par le médecin du travail sert à dédouaner le poste de travail et contribue à faire porter la responsabilité de la maladie sur l'individu.

\section{Le rôle du médecin, entre consultations individuelles et tiers temps}

C'est en 1979 que le «tiers temps» devient obligatoire pour remplir une fonction de prévention, essentiellement primaire, sur les lieux de travail, afin de mesurer les risques pour la santé, principalement physique. Avec le temps, la notion de santé au travail s'élargit, reconnaissant la souffrance psychique pouvant être causée par l'organisation du travail. Cette catégorie, dénommée «souffrance au travail », s'appuie sur l'activité clinique du médecin du travail et le dote d'un nouveau mandat où il devra être plus attentif au vécu subjectif des travailleurs (Salman, 2008).

Par la suite, le médecin se trouve confronté à des conflits de plus en plus importants dans l'exercice de son activité. Par exemple, dans les années 19851990, alors que la crise de l'emploi s'accentue, la délivrance d'un avis d'aptitude négatif peut conduire le salarié à perdre son travail (Huez, 1998). Les médecins du travail se trouvent alors dans une tension forte entre «droit au travail» et «droit à la santé ». De plus, l'État s'attend à ce que le médecin du travail atteignent les objectifs et les exigences attendus en matière de préventions primaire, secondaire et tertiaire dans le cadre d'approches individuelles (consultations) et collectives (actions en milieu de travail).

\section{En réponse à la pénurie de médecins et à la nécessité d'améliorer} la prévention, la pluridisciplinarité est imposée

La Loi de modernisation sociale (du 17 janvier 2002) prévoit la pluridisciplinarité pour les entreprises et les services de santé au travail, alors que cette 
dernière avait déjà été énnoncée dans la Convention de l'OIT n¹61 de 1985. Depuis 2004, plusieurs réformes se succèdent, prônant davantage de pluridisciplinarité comme voie pour une prévention plus efficace des risques professionnels (Aubin et al., 2007; Dellacherie et al., 2010). Les «services de médecine » ont disparu au profit des «services de santé au travail » (SST), avec l'embauche d'intervenants en prévention des risques professionnels (IPRP). Ces réformes peuvent être analysées comme le résultat d'une action patronale (Marichalar, 2010) débutée dans les années 1970 qui visait à réduire l'autonomie des médecins et à diminuer les coûts de la prévention. L'objectif est de passer de l'approche individuelle centrée sur le cabinet médical à l'approche collective de terrain. Ce nouveau contexte oblige les médecins à travailler avec d'autres professionnels provenant de diverses disciplines (ingénieurs sécurité ou spécialistes en risques professionnels, ergonomes, psychologues du travail, kinésithérapeutes, ergothérapeutes, etc.). Si certains médecins du travail s'étaient déjà engagés, bien avant la réforme, dans des actions multiprofessionnelles (Boitel, 2006), les nouvelles lois exigent de mettre en œuvre la pluridisciplinarité afin d'améliorer l'efficacité de la prévention. Par ailleurs, la figure de la médecine du travail en France évolue à cause d'une démographie vieillissante qui ne permettra plus de tenir le métier de la même façon (Caillard, 2011). De plus, l'émergence de nouveaux métiers dans le champ de la prévention, tels que ceux d'infirmière de santé au travail (IST) et d'assistant de santé au travail (AST), obligent les médecins du travail à repenser leurs territoires de compétences et les complémentarités entre les professionnels, notamment en matière de prévention des risques professionnels complexes comme les TMS.

La Loi du 20 juillet 2011 donne au médecin du travail un rôle de coordination (L.4622-8) de l'équipe pluridisciplinaire. Cette réforme conduit aussi à une surveillance médicale renforcée de certaines populations au travail considérées plus exposées à des risques qui sont reconnus par la législation ${ }^{3}$ comme pouvant porter atteinte à la santé. Peu de temps après, la Loi de 2016 vient marquer un renforcement du rôle du «médecin coordinateur » et une activité de consultation orientée vers le suivi de population en difficultés de santé par les médecins du travail. Le contexte législatif donnera ici une autre posture prescriptive aux médecins dans le domaine de la prévention et des relations avec les autres professionnels.

Retenons donc qu'au départ, en France, la pluridisciplinarité n'était pas inscrite dans le cadre législatif comme moyen de faire de la prévention. Par la suite, la prévention, portée initialement par le médecin du travail, s'appuiera dorénavant sur un travail pluridisciplinaire que le médecin coordonne. Toutefois, comme nous le verrons, ceci n'ira pas sans poser de difficultés dans la réalisation du travail collectif. 


\section{Comparaison des contextes des dispositifs de prévention Québec/France}

En France, comme au Québec, l'élaboration du régime de santé au travail a été guidée par la volonté des États de sortir de la «médecine d'entreprise» (Ferré, 2010). Cependant, les services ont connu des évolutions différentes puisqu'en France, on est passé d'une mission axée essentiellement sur les visites médicales individuelles à une mission complémentaire d'action en milieu de travail, pratique qui s'est davantage affirmée avec l'introduction de la pluridisciplinarité. Au Québec, I'approche de santé publique pluridisciplinaire est en place depuis le début, mais l'État n'a pas rendu les services accessibles à tous les milieux de travail. De plus, les moyens octroyés aux ESAT plafonnent, alors que le nombre d'établissements à desservir augmente (Plante, Bherer et Vézina, 2010). En France, la pluridisciplinarité existait déjà implicitement dans les pratiques de certains médecins du travail. Toutefois, elle a été formalisée plus récemment, traduisant la volonté de l'État de rendre plus efficaces les actions de prévention en milieu de travail, cela, dans un contexte de pénurie de médecins du travail. Cette pénurie est d'ailleurs l'un des importants défis auxquels doivent faire face tant la France que le Québec.

\section{Les interventions de prévention des TMS et le travail collectif pluridisciplinaire}

Les TMS constitue un exemple de problème de santé dont la prévention exige une approche pluridisciplinaire. En effet, les connaissances étiologiques démontrent aujourd'hui que leur apparition est liée à de nombreux facteurs de risque (Côté et al., 2013; Aptel et al., 2011) de nature bio-mécanique (postures, efforts, vibrations) et aussi psycho-sociale (organisation du travail, degré de latitude, exigences), auxquels s'ajoutent des facteurs personnels. Ainsi, pour détecter et agir sur ces multiples facteurs combinés dans une situation de travail exposant les salariés, il convient de recourir à des approches pluridisciplinaires. Selon Vinck (2000, 2002), la pluridisciplinarité correspond à la co-présence de plusieurs disciplines, c'est-à-dire que plusieurs professionnels issus de disciplines différentes concourent à un même objectif en étant amenés à travailler ensemble. Comme nous le verrons lors de la présentation du cadre conceptuel de notre analyse, différentes formes de travail collectif ont été décrites du point de vue de l'ergonomie. Cependant, les situations de travail mettant en jeu des professionnels de disciplines différentes ont été peu examinées sous cet angle. Au surplus, le rôle du contexte comme influençant spécifiquement le travail collectif lors des interventions n'a pas fait, non plus, l'objet d'attention. C'est ce que nous proposons d'examiner dans cet article. 


\section{Cadre conceptuel et questions de recherche}

Nous nous intéresserons ici au travail collectif pluridisciplinaire (TCP), c'est-àdire au travail dans lequel se côtoient des professionnels provenant de différentes disciplines et ayant pour mission la prévention des risques complexes dans un milieu de travail. En ergonomie, différentes formes de travail collectif ont été décrites. C'est ainsi que De la Garza et Weill-Fassina (2000) les ont définies selon la nature des interactions sociales. Ces auteurs distinguent quatre types, soit coaction, coopération, collaboration et entraide, proposant une sorte de contiunum vers des formes de travail collectif de plus en plus évoluées. Des études plus récentes en ergonomie (Caroly, 2010) ont aussi montré que la collaboration constitue, plus qu'un travail collectif, une véritable activité collective qui contribue à l'efficacité productive et à la santé des personnes qui y participent. Cette typologie fait écho à d'autres travaux sur la collaboration interprofessionnelle dans le domaine des sciences infirmières (D'Amour et al.,2005; Fortier, Brahimi et Fortier, 2013), notamment pour décrire les pratiques de collaboration interprofessionnelle ( $\mathrm{PCl}$ ) des équipes de soins et faire un lien avec l'efficacité des interventions. L'ensemble de ces auteurs suggèrent que les formes les plus évoluées de $\mathrm{PCl}$ donnent lieu à des interventions plus efficaces.

Pour les fins de notre étude, nous avons retenu deux formes de travail collectif en matière de prévention des risques, soit: 1-la co-action, qui concerne des situations dans lesquelles des professionnels réalisent des tâches différentes sur des objets différents avec des buts à court terme différents, mais pouvant s'intégrer à long terme dans une action commune (Savoyant, 1977); et 2-la collaboration, qui est présente lorsque des professionnels accomplissent des actions sur un même objet en partageant des buts à court et moyen termes (Rogalski, 1994; Caroly, 2010). Les deux formes retenues, en plus de se distinguer nettement sur le plan empirique, représentent respectivement une forme de TCP «simple», la co-action, et une plus «évoluée», la collaboration.

Nous tenterons de répondre aux questions de recherche suivantes: 1-Quelles sont les modalités du TCP lors des interventions de prévention des TMS dans chacun des contextes; 2-Comment se manifestent les formes de TCP que sont la co-action et la collaboration lors des interventions; 3-En quoi les contextes respectifs de la France et du Québec constituent des ressources ou des obstacles pour le TCP dans les interventions externes de prévention des TMS.

\section{Méthodologie}

Afin de documenter les pratiques réelles d'intervention sur les TMS, des données empiriques ont été recueillies à l'aide de différentes méthodes. La méthodologie est exposée ici dans ses grandes lignes. Les détails peuvent être 
obtenus dans des publications antérieures (Caroly et al. 2016) pour les études réalisées en France (Caroly, 2013) et au Québec (Prud'homme, Bellemare et Baril-Gingras, 2011).

\section{Au Québec}

Quatorze intervenants volontaires (médecins du travail, infirmières, techniciens en hygiène du travail, ergonomes) appartenant à six ESAT, provenant de trois régions ${ }^{4}$ différentes ont participé à l'étude. Une partie des données a été recueillie par entretiens individuels semi-dirigés, variant de 30 à 90 minutes, auprès de dix d'entre eux. De plus, deux entretiens d'auto-confrontation à partir des traces écrites de l'activité (rapports, dossiers d'entreprises, PSSE) ont été réalisés, dans le but de reconstituer a posteriori les interventions réalisées. Quatre observations directes ont aussi été effectuées, avec prises de notes ou enregistrement audio, dont trois en entreprise et une dans les bureaux de l'ESAT. II s'agissait de deux rencontres avec un milieu pour le renouvellement du PSSE, auxquelles participaient un médecin, une infimière et un technicien; d'une évaluation ergonomique d'un poste de travail menée par le médecin et l'infirmière; d'une réunion d'un groupe de travail regroupant différents intervenants des ESAT d'une région, pour débattre des enjeux et des problèmes rencontrés sur le terrain autour de la prévention des TMS. Les entretiens et les observations ont été audio-enregistrées et transcrites ou ont fait l'objet de prise de notes. Finalement, une synthèse des données sur les pratiques réelles d'intervention de prévention des TMS a été présentée dans deux des régions participantes, de même que lors d'une rencontre réunissant l'ensemble des ergonomes du Réseau de santé publique en santé au travail pour validation.

\section{En France}

Des entretiens et des observations ont été réalisés dans dix SSTi, répartis sur le territoire (régions Rhône-Alpes, Bordeaux, Paris), auxquels ont participés 43 intervenants volontaires (médecins du travail-MDT, assistants de santé au travailASST, intervenants de prévention des risques professionnels-IPRP et infirmières de santé au travail-IDEST). Plus précisément, 21 entretiens individuels ont été réalisés auprès des intervenants afin de comprendre les pratiques professionnelles collectives selon le métier de chacun et les formes de pluridisciplinarité dans les interventions de prévention des TMS. En ce qui a trait aux observations du travail des équipes sur le terrain dans le cadre des TMS, neuf séquences d'observation ont été réalisées: trois IPRP-MDT (visite en entreprise, étude ergonomique); deux ASST-MDT (évaluation des risques TMS dans une très petite entreprise de coiffure, visite en entreprise); et quatre IDEST-MDT (restitution d'entretien infirmier à propos de TMS). À I'issue de ces observations, nous avons réalisé six entretiens d'auto-confrontation simple (un IPRP, un AAST, deux IDEST, deux MDT) et trois 
entretiens d'auto-confrontation croisée (un binôme AST-MDT et deux binômes IDEST-MDT). Nous avons effectué également quatre réunions collectives, une avec chacun des métiers présents dans les services de santé au travail.

\section{Les modalités du travail collectif pluridisciplinaire en prévention des TMS}

\section{Au Québec, une intervention prescrite, que les équipes adaptent localement}

\section{Une approche structurée face aux TMS, mise en œuvre collectivement}

Tel que mentionné précédemment, le travail des ESAT consiste à élaborer un programme de prévention spécifique à l'établissement (PSSE) dans lequel seront identifiés, en ordre de priorité, les différents risques auxquels les travailleurs sont exposés. Lorsqu'un établissement lui est attribué, l'équipe d'intervention constitue un dossier sous forme de fichier électronique, dans lequel sont consignées les informations-clés sur l'entreprise (secteur d'activité économique, type de production, effectifs, coordonnées des interlocuteurs, etc.). Cet outil est accessible à tous les membres de l'ESAT qui peuvent alors le consulter et l'alimenter. Lors d'une première visite, à laquelle participent au moins deux intervenants, (par exemple, le médecin et l'infirmière ou le technicien et l'infirmière), la direction est rencontrée, de même que le représentant des travailleurs. Puis, les membres de l'équipe inspectent les lieux afin de repérer la présence de différents risques et de les consigner, le cas échéant, dans le PSSE. Cette visite est aussi I'occasion de vérifier si l'entreprise est en mesure d'offrir les premiers soins et les premiers secours (PSPS) en cas de blessures. C'est l'infirmière qui se charge de cette tâche.

En ce qui a trait aux TMS, les membres des ESAT ont tous reçu une même formation et appris à utiliser différents outils de dépistage auxquels ils ont recours lors de la visite de l'établissement. La décision de retenir ou non le risque TMS dans le PSSE est prise collectivement, comme c'est le cas d'ailleurs pour les autres risques. II faut noter qu'en marge de cette tâche technique de dépistage des risques, les intervenants mènent une tâche parallèle qui consiste à convaincre l'entreprise de la nécessité d'agir en prévention. Souvent, en effet, les entreprises desservies ne sont pas du tout sensibilisées à la santé au travail et encore moins demandeuses d'intervention de prévention des TMS. Les intervenants sont ainsi amenés, au travers des échanges avec les différents acteurs du milieu, à déployer un argumentaire destiné à convaincre les employeurs, et parfois les travailleurs eux-mêmes, des risques présents et des actions qui s'imposent pour préserver la santé des salariés. D'autres outils ont donc été développés dans le Réseau de santé publique en santé au travail afin de susciter une prise de conscience des acteurs de l'entreprise face aux risques de TMS. 


\section{Un travail collectif pluridisciplinaire que les équipes adaptent localement}

Même si la manière de traiter le risque TMS est prescrite et s'appuie sur des outils issus de I'Institut national de santé publique du Québec (INSPQ), organisme responsable d'évaluer l'efficacité du programme de prévention des TMS, il s'avère que les pratiques réelles sont diverses, notamment en ce qui a trait au rôle dévolu à chacun des intervenants (Prud'homme, 2011). Le médecin est responsable, de par la loi, de l'élaboration du PSSE. Cependant, le faible nombre de médecins fait en sorte que, dans les faits, les techniciens et les infirmières sont très impliqués dans I'élaboration du PSSE et des activités qui en découlent. Lorsque le risque TMS est retenu au PSSE, le déroulement de l'intervention varie, selon ce que conviennent entre eux les membres de l'équipe d'intervention. Ainsi, le repérage des risques peut être fait par l'un ou l'autre des trois membres de l'équipe. Le plus souvent, c'est l'infirmière qui le fera et, parfois, le technicien. La session d'information sur le risque TMS peut être donnée dans le milieu par l'infirmière ou le technicien. En ce qui concerne l'ergonome, il ne fait pas partie de l'équipe d'intervention, mais il peut être appelé à participer en diverses occasions afin de: 1-valider le repérage des risques, si l'équipe le juge opportun; 2-prendre en charge la rencontre d'information dans le milieu, ou y participer; 3-approfondir le diagnostic; 4-accompagner le milieu dans la recherche de solutions.

Lors de leurs interventions, les équipes ont recours à l'outil " perception du milieu » qui leur permet de s'appuyer sur les préoccupations des acteurs de l'établissement afin de cibler un poste-clé où des actions de changement sont jugées pertinentes. Cet outil permet alors une délibération collective dans le but de décider des modalités de l'intervention et il aide l'équipe à être perçue comme une ressource pour l'entreprise.

\section{En France, une pratique peu prescrite, décidée par le médecin et pouvant faire l'objet de délégation}

\section{Un travail collectif pluridisciplinaire peu prescrit}

Dans le contexte français, les actions en milieu de travail, coordonnées par le médecin du travail qui a la responsabilité du suivi de l'entreprise et de ses salariés, sont peu formalisées. Elles dépendent des compétences spécifiques de chacun des professionnels et de leur capacité à travailler ensemble. Les actions menées sont diversifiées, et les approches, les outils varient selon les personnes, leur formation et l'organisation du SSTi.

Les actions collectives pluridisciplinaires en milieu de travail peuvent être influencées par le «projet de service». Ce document, obligatoire pour l'accréditation des SSTi, doit préciser les axes prioritaires de prévention et les moyens 
mis en œuvre dans le service pour y arriver. Parmi ces moyens, on peut retrouver le développement d'outils visant à faciliter le travail collectif pluridisciplinaire comme: 1-la fiche d'entreprise, réalisée par le médecin du travail, avec l'aide de I'IPRP ou de I'AST; 2-des outils informatiques de cueillette de données sur la santé des salariés par le médecin ou l'infirmière qui orientent des actions de prévention par branches professionnelles ou types de risque. Pour le risque TMS, une grille de cotation des manutentions manuelles dans les entrepôts, élaborée par les IPRP en concertation avec les médecins, devient un support d'évaluation du risque TMS pour les autres professionnels du service de santé au travail. Cette approche plutôt biomécanique des TMS peut tout de même amener les professionnels à réfléchir sur les actions à engager du côté de l'organisation du travail comme moyen de prévention primaire.

Penser et mener une action en milieu de travail en réponse à une demande

Plusieurs des actions en milieux de travail sont initiées à la suite de consultations médicales ou d'entretiens infirmiers. La pratique de la pluridisciplinarité en France s'organise principalement autour du médecin qui reçoit dans son cabinet des salariés en souffrance. C'est donc à partir de la consultation médicale individuelle qu'aura lieu, la plupart du temps, une action en milieu de travail. Par exemple, l'alerte sera donnée en CHSCT par le médecin du travail sur l'usage d'équipement contraignant pour le dos. Ceci peut être l'occasion d'engager un travail collectif avec les autres professionnels du service de santé au travail. C'est ainsi que le médecin peut déléguer une partie des actions en milieu de travail aux IPRP, en fonction de leurs compétences notamment, en ce qui a trait aux TMS, à des ergonomes (diagnostics au poste, recherche de solutions pour l'entreprise, accompagnement).

De plus, chaque salarié a la possibilité, entre deux visites médicales périodiques, de rencontrer l'infirmière du travail lors d'un entretien particulier, appelé «entretien infirmier». Les informations transmises au médecin par l'infirmière concernant les plaintes des salariés ou les risques présents amènent, dans certains cas, le médecin, en concertation avec l'infirmière, à décider d'agir en milieu de travail (le médecin seul, I'infirmière seule ou les deux). II peut proposer, entre autres, à l'entreprise une sensibilisation des salariés sur les risques TMS et les moyens de se protéger, en organisant des rencontres de groupe sur les heures de travail, rencontres animées par l'infirmière. D'ailleurs, depuis l'arrivée des infirmières dans les services de santé au travail, les médecins peuvent discuter avec elles des dilemmes qu'ils peuvent rencontrer dans leur activité (par ex. : préservation de la santé vs protection de l'emploi du salarié) et se sentir moins seuls dans les actions à engager pour l'accompagnement du salarié ou de l'entreprise. 
Parfois, c'est l'entreprise (par exemple, le CHSCT ou la DRH) qui formule directement une demande au médecin pour une action en milieu de travail concernant les TMS. Dans ce cas, le médecin du travail, qui est lui-même porteur de pluridisciplinarité, convoque l'action d'un professionnel d'une autre discipline (par exemple, en ergonomie, psychodynamique du travail, épidémiologie, etc.) pour répondre à la demande de l'entreprise. Par ailleurs, face aux difficultés de convaincre les employeurs ou d'aider le salarié à résoudre le problème, certains médecins n'hésitent pas, pour trouver des issues favorables à la prévention, à collaborer avec d'autres acteurs dans l'entreprise (direction des ressources humaines, par exemple) ou avec des partenaires externes (inspecteur du travail, assistante sociale ou psychologue, conseiller de prévention de la sécurité sociale ou ergonome en cabinet, etc.).

\section{Comparaison des formes du travail collectif pluridisciplinaire}

Le tableau 1 résume et compare les modalités du TCP lors des interventions de prévention des TMS dans les contextes du Québec et de la France.

Nous retiendrons qu'en France la pluridisciplinarité, instaurée récemment, se traduit par du travail collectif qui passe obligatoirement par le médecin, lequel délègue une partie de la tâche que, jusque-là, il était le seul à pouvoir accomplir. Le travail se décline en binôme et le médecin y est souvent présent. Le TCP

TABLEAU 1

Modalités du travail collectif pluridisciplinaire (TCP) en prévention des TMS

\begin{tabular}{lll}
$\begin{array}{l}\text { Décision de mener } \\
\text { une intervention }\end{array}$ & $\begin{array}{l}\text { Au Québec } \\
\text { Prise par l'équipe grâce à la consignation } \\
\text { du "risque TMS" dans le PSSE }\end{array}$ & $\begin{array}{l}\text { En France } \\
\text { Prise par le médecin, } \\
\text { en lien avec des demandes exprimées } \\
\text { directement ou indirectement }\end{array}$ \\
\hline $\begin{array}{l}\text { Modalités } \\
\text { de l'intervention }\end{array}$ & $\begin{array}{l}\text { Prescrites, transmises (lors d'une formation } \\
\text { commune) et adaptées par l'équipe } \\
\text { selon les particularités de l'entreprise }\end{array}$ & $\begin{array}{l}\text { Peu prescrites, déterminées } \\
\text { par le médecin selon la formation } \\
\text { qu'il a reçue }\end{array}$ \\
\hline $\begin{array}{l}\text { Répartition } \\
\text { des rôles }\end{array}$ & $\begin{array}{l}\text { Chaque membre de l'équipe } \\
\text { peut participer à l'intervention, mais } \\
\text { lorsqu'un ergonome fait partie de l'ESAT, } \\
\text { I'on a recours à son expertise }\end{array}$ & $\begin{array}{l}\text { Le médecin peut déléguer des actions } \\
\text { aux IPRP (évaluation des risques), } \\
\text { aufirmières (séances d'information }\end{array}$ \\
\hline $\begin{array}{l}\text { Outils } \\
\text { et des acteurs hors de son service }\end{array}$ \\
\hline $\begin{array}{l}\text { Répertoire commun avec possibilités } \\
\text { de choisir localement }\end{array}$ & $\begin{array}{l}\text { Peu d'outils formalisés partagés } \\
\text { entre acteurs }\end{array}$ & $\begin{array}{l}\text { Échanges d'information, } \\
\text { décisions prises ensemble }\end{array}$ \\
\hline $\begin{array}{l}\text { Formes du TCP } \\
\text { Le plus souvent, collaboration entre } \\
\text { les membres de l'équipe d'intervention; } \\
\text { co-action de l'ergonome avec l'équipe } \\
\text { d'intervention }\end{array}$ & $\begin{array}{l}\text { Échanges d'information et délégation } \\
\text { de la part du médecin }\end{array}$ \\
\hline
\end{tabular}


médecin/IPRP prend la forme d'une co-action ou d'une collaboration. II peut prendre, parfois, la forme de coopération ou d'entraide lorsque le binôme infirmière/médecin ou IPRP/médecin travaillent ensemble depuis un certain temps et qu'ils partagent des références communes sur la manière de faire la prévention des TMS dans l'entreprise et auprès du salarié.

Au Québec, le TCP réalisé par les équipes d'intervention en matière de prévention des TMS est fortement encadré. II l'est, à la fois par des règles institutionnelles qui, dès le départ, ont déterminé la composition pluridisciplinaire des ESAT et par la prescription d'une démarche structurée, décidée institutionnellement et comportant des outils que les intervenants ont pu s'approprier à travers une formation commune. Ce référentiel partagé nous semble permettre des formes plus élaborées de travail collectif dans les équipes d'intervention, avec des délibérations pour prendre des décisions concertées. Par ailleurs, comme les entreprises sont, le plus souvent, non demandeuses d'intervention en santé au travail, cela oblige les équipes à recourir à des stratégies pour convaincre l'entreprise de la pertinence de faire de la prévention. Ainsi, la préoccupation partagée par tous les professionnels de sensibiliser les milieux et de les amener à se prendre en charge, comme le préconise l'approche de santé publique, donne lieu à des formes de travail collectif pouvant aller jusqu'à la coopération et l'entraide (Caroly, 2013). Notons que le travail collectif avec l'ergonome, qui s'avère être plutôt une ressource professionnelle que l'équipe d'intervention peut appeler au besoin, s'apparente davantage à de la co-action.

\section{Ressources et contraintes du contexte pour le travail collectif pluridisciplinaire au Québec et en France}

Étant donné que les actions de prévention des TMS donnent lieu à des formes différentes de TCP au Québec et en France, nous discuterons maintenant des éléments du contexte considérés soit comme ressources, soit comme contraintes pour le TCP dans ces deux endroits.

\section{Des ressources pour le travail collectif pluridisciplinaire}

La souffrance entendue en consultation: un soutien au travail collectif et à l'élargissement du périmètre d'action

Dans le contexte français, on remarque que les actions en milieu de travail s'avèrent, le plus souvent, une réponse pour apaiser la souffrance des travailleurs et une tentative de prévenir des atteintes à d'autres salariés. L'activité clinique apparaît ici comme une ressource pour la prévention primaire : elle crée une sorte de référentiel commun sur la souffrance au travail. Le fait que le médecin établisse le diagnostic médical de TMS dans sa consultation représente un appui solide 
pour réclamer des changements aux postes de travail. Ainsi, l'intégration des missions de prévention primaire, secondaire et tertiaire existant dans le système français, avec une activité clinique exercée par le médecin et par l'infirmière, permet de construire une demande qui soutient l'engagement éventuel des autres professionnels dans les actions qui visent à prévenir les risques, particulièrement lorsqu'il s'agit de risques complexes. Ceci n'est pas sans rappeler la mobilisation subjective, l'engagement dans une œuvre commune qui, pour Dejours (1993), constitue une condition de la coopération.

De plus, la parole des salariés entendue en cabinet par le médecin permet d'orienter les actions en milieu de travail, au-delà des facteurs biomécaniques, vers les facteurs de risque reliés à l'environnement psycho-social du travail. Les mal-être exprimés en consultation peuvent guider l'action de prévention vers des changements touchant I'organisation du travail, les relations sociales dans l'entreprise, particulièrement lorsque le médecin a acquis une formation sur les RPS et que les autres professionnels sont formés à ce type d'approche.

Au Québec, le médecin et l'infirmière n'ont pas accès formellement à l'expression de la souffrance des salariés et ceci pourrait expliquer la raison pour laquelle les équipes disent craindre parfois que le risque TMS ne soit pas jugé "assez grave» (Stock, Tissot et Lazreg, 2014) pour exiger que l'entreprise investisse dans le but de le contrer. La parole des salariés est accessible, mais indirectement, à travers celle de leurs représentants ou des acteurs décisionnaires, ou encore grâce à l'administration de questionnaires. De plus, les entreprises desservies, concentrées dans les secteurs prioritaires, sont aux prises avec des risques traditionnels (exposition aux poussières, aux produits chimiques, au bruit), ce qui peut conduire à faire paraître le risque TMS peu important. Les secteurs reconnus comme les plus à risques de ce point de vue, notamment celui de la Santé et des services sociaux, ne font pas partie des secteurs priorisés actuellement par l'État. Les ESAT sont donc souvent confrontées à des entreprises qualifiées de "récalcitrantes» (Tougas, 2014) en matière de prévention des TMS.

\section{Une mission commune, une démarche d'intervention structurée avec des outils partagés}

Au Québec, la mission des ESAT est de faire en sorte que l'entreprise prenne en charge la prévention, que celle-ci soit l'objet d'une préoccupation constante à travers toutes ses activités productives. Voilà donc un objectif auquel chacun des acteurs de l'équipe se doit de contribuer. Ainsi, au-delà des compétences techniques propres à chacun des métiers, les membres de l'équipe partagent des compétences stratégiques (Prud'homme, 2019) qui s'avèrent également favorables à des formes poussées de TCP. L'approche de santé publique mise en œuvre 
par les équipes d'intervention suppose une démarche structurée de connaissance de l'entreprise elle-même, qui permet d'élaborer des stratégies tenant compte des capacités et des dispositions des acteurs à agir en prévention.

Comme le rapporte Vézina (2010), la diminution de la prévalence des incapacités liés aux troubles musculosquelettiques constitue l'un des objectifs du Programme national de santé publique 2003-2012. La démarche de prévention des TMS, mise en œuvre par les ESAT au Québec, a été pensée globalement et s'inscrit dans le cadre institutionnel de la santé publique, bénéficiant des ressources de I'INSPQ et des contributions d'acteurs du réseau via une table de concertation. Elle a été conçue à la lumière d'un état des connaissances scientifiques, avec des outils considérés comme ayant fait leur preuve. Les intervenants des trois métiers représentés dans les équipes d'intervention ont pu s'approprier la démarche proposée à travers une formation commune. Ce référentiel commun constitue certainement une ressource importante pour le travail collectif, notamment grâce à sa dimension "opérative», comme le suggèrent Terssac et Chabaud (1990). |l ouvre la porte à des formes de TCP plus poussées où, par des régulations essentiellement horizontales, les intervenants sont amenés à coopérer et à s'entraider. II semble que la présence d'ergonomes «augmente significativement la quantité d'interventions en prévention des TMS, de même que la diversité des pratiques de soutien en milieu de travail » (Stock et al., 2006: 18). Or, les ergonomes ne font pas partie systématiquement des ESAT et, selon les intervenants, il s'agit d'un élément-clé pour favoriser la prévention des TMS dans les entreprises (Stock, Tissot et Lazreg, 2014).

Contrairement au Québec, I'approche de la prévention des TMS en France est relativement peu formalisée par des démarches ou outils communs. Seules quelques actions en prévention tertiaire, de type maintien dans l'emploi, sont un peu formalisées dans leur dimension clinique (visite de reprise, mi-temps thérapeutique, aménagement de poste). On observe plutôt des formes de TCP de type co-action ou collaboration, dans lesquelles on retrouve des régulations verticales où le médecin, acteur principal, plus traditionnellement reconnu pour assurer la prévention, fait appel à des collaborateurs de son service et, quelquefois, de l'extérieur de celui-ci afin de leur octroyer des mandats.

\section{Des collectifs de métier à l'appui du travail collectif pluridisciplinaire}

Au Québec, il existe, au sein du réseau de la santé publique en santé au travail, des regroupements par métiers. C'est ainsi que l'on note la présence d'une «Table des représentants régionaux en ergonomie, en hygiène ». Bien qu'aucune étude n'ait examiné les activités de ces groupes, on peut penser qu'ils constituent des lieux privilégiés pour parler du métier et, en cela, ils peuvent alimenter la pratique du travail collectif (Caroly, 2010; Clot, 2006). 
La tension entre les volets clinique, d'une part, et action en milieu de travail, d'autre part, amène parfois le médecin du travail français à devoir réaliser seul certains arbitrages bien que, dans plusieurs services, des dispositifs soient en place pour discuter du métier. Pourtant, la construction de ressources dans I'intra-métier pourraient soutenir le travail inter-métiers (Poussin, 2016).

\section{Des contraintes pour le travail collectif ou le travail collectif empêché}

\section{Les consultations individuelles, frein possible aux actions en milieu de travail}

Paradoxalement, les activités de consultation réalisées par les médecins du travail en France peuvent empêcher le travail collectif, puisque celui-ci se déploie surtout dans les actions de prévention en milieu de travail. Certains services peuvent prioriser, plus ou moins formellement, des critères de performance autour du nombre de visites et réduire ainsi les actions en milieu de travail. On peut remarquer également que les professionnels qui entourent les médecins peuvent se retrouver empêchés d'exercer un rôle significatif en milieu de travail. Ainsi, il semble que malgré leur formation, les infirmières de santé au travail sont plutôt cantonnées dans les activités d'entretien infirmiers, ou les IPRP au recensement des expositions professionnels dans le cadre de la rédaction de la fiche d'entreprise.

\section{L'offre de service restreinte}

Au Québec, le fait que les milieux de travail classés «non prioritaires » n'aient pas accès aux services empêche les équipes d'aller plus loin avec des entreprises qui, pourtant, seraient prêtes à s'attaquer aux risques complexes et qui ont grandement besoin de ressources. De plus, le programme d'intervention ne prévoit pas, non plus, d'accompagner les entreprises dans leurs projets, alors que I'on sait qu'il s'agit bien d'une modalité importante de la prévention durable (Caroly et al., 2008).

\section{Le statut prépondérant du médecin du travail}

Le fait que le médecin agisse comme coordonnateur de l'équipe pluridisciplinaire lui confère un statut particulier qui l'amène à procéder par délégation, et à se situer "au-dessus » de l'équipe, ce qui apparaît moins favorable au TCP. La sociologie des professions américaine s'est penchée sur cette question de la coopération entre métiers ou professions (au sens de profession établie disposant d'un monopole d'exercice), particulièrement dans le domaine de la santé. Elle a fait ressortir les enjeux identitaires de toute situation de pluridisciplinarité, enjeux qui se jouent tant sur le plan institutionnel que sur le plan de la coopération concrète (Cholez et al., 2011). 


\section{Conclusion}

Nos données ne sont pas exhaustives, mais en prenant pour objet les interventions de prévention des TMS dans deux États, et en s'appuyant sur un travail de terrain, cette recherche nous amène à explorer le rôle du contexte "macro » dans les modalités d'intervention en milieu de travail. L'angle du travail collectif nous permet de développer un nouvel objet pour l'ergonomie: le TCP et son rôle dans l'efficacité des interventions, interventions qui ont de plus en plus pour objet des «risques complexes ». De plus, nos données suggèrent que les catégories utilisées pour décrire les formes du travail collectif pourraient être développées, cela afin de mieux prendre en compte la dimension pluridisciplinaire lorsqu'elle est présente. Des travaux récents (Prud'homme, 2019) montrent, en effet, que des collectifs de travail pluridisciplinaires efficaces ont recours, à certains moments de leurs interventions, à du travail collectif de type co-action.

De plus, la comparaison entre nos deux systèmes de santé au travail invite à s'interroger sur les orientations futures à leur donner. Du côté québécois, l'une des conditions de l'efficacité de la prévention serait d'étendre la couverture limitée actuellement aux entreprises des secteurs prioritaires, à d'autres secteurs d'activités économiques où les risques de TMS et les RPS sont présents, notamment celui de la Santé et des services sociaux où les acteurs sont demandeurs d'action de prévention à la source. En ce moment, les équipes d'intervention se retrouvent concentrées dans des entreprises qui ne sont pas nécessairement prêtes à agir pour prévenir ces risques. De plus, la présence des ergonomes semble une condition favorisant la prévention durable, et leur participation pourrait s'actualiser à travers un accompagnement de l'entreprise.

En France, il nous semble que les réformes pourraient davantage prendre en compte l'activité de consultation individuelle à titre de ressource dans l'activité de prévention des médecins du travail, tout en favorisant les moyens de travail collectif horizontal avec l'entreprise et le travail en réseau pluri-professionnel Ceci apparait d'ailleurs dans le dernier Rapport Lecoq (2018) sur la restructuration du dispositif de santé au travail en France. Pour l'avenir de la prévention, il appert important de permettre les différentes formes de prévention chez les médecins du travail: préventions individuelles et collectives, mais, aussi, d'inclure la diversité des métiers et leurs rôles dans la prévention durable, en respectant les compétences et les identités professionnelles, tout en renforçant la coordination intermétiers. 


\section{Notes}

1 Cet organisme est, depuis le premier janvier 2016, la Commission des normes, de l'égalité, de la santé et de la sécurité du travail (CNESST).

2 II est important de noter que les "médecins responsables» ont des fonctions autres que celles reliées aux PSSE, mais qu'ils ne font pas de consultations individuelles systématiques, ni d'évaluation de l'aptitude des travailleurs, ni d'examens d'embauche.

3 II s'agit d'une consultation médicale avec examen systématique, souvent annuel, pour des populations exposées à des risques reconnus par le Code du travail pour leur pénibilité (ex.: horaire atypique, risque CMR, etc.), alors que les autres salariés pourront avoir une visite médicale systématique avec le médecin du travail tous les 3 ou 4 ans, ainsi qu'un entretien infirmier aux 2 ans environ.

4 Afin de respecter les engagements de confidentialité, les régions ne sont pas nommées. II s'agit d'une région urbaine et de deux régions semi-urbaines.

\section{Références}

Aptel, Michel, François Cail et Agnès Aublet-Cuvelier (2011) Les troubles musculosquelettiques du membre supérieur (TMS-MS). Guide pour les préventeurs, Québec: Édition de I'Institut national de recherche et de sécurité (INRS), ED 957, 2e édition.

Aubin, Claire, Régis Pélissier, Pierre De Saintignon, Jacques Veyret, Françoise Consot et Paul Frima (2007) Rapport sur le bilan de réforme de la médecine du travail. Paris : IGAS. [En ligne] « https://www.ladocumentationfrancaise.fr/var/storage/rapports-publics/074000708. pdf».

Baril-Gingras, Geneviève, Marie Bellemare et Jean-Pierre Brun (2006) «Interventions externes en santé et en sécurité du travail: influence du contexte de l'établissement dans l'implantation de mesures préventives », Relations industrielles/Industrial Relations, 61 (1), 9-41.

Baril-Gingras, Geneviève, Marie Bellemare et Chantal Brisson (2012) «How Can Qualitative Studies Help Understand the Role of Context and Process of Interventions on Occupational Safety and Health and on Mental Health at Work?», dans C. Biron, M. Karanika-Murray et C. L. Cooper, Organizational Stress and Well-being Interventions: Addressing Process and Context, Londres: Psychology Press, p. 135-162.

Berthelette, Diane et Raynald Pineault (1992) «Analyse d'implantation du programme de santé au travail. Résultats d'une recherche évaluative», Travail et santé, 8 (4), S23-30.

Berthelette, Diane (2002) «Les déterminants de l'implantation et des résultats des programmes de santé et de sécurité du travail », dans Denis Harrisson et Camille Legendre, Santé, sécurité et transformation du travail. Réflexions et recherche sur le risque professionel, Sainte-Foy: Les Presses de I'Université du Québec à Montréal, p. 107-128.

Boitel, Lilianne (2006) « Mise en place de la pluridisciplinarité en santé au travail. État des lieux dans les entreprises ", Archives des maladies professionnelles et de l'environnement, 67 (2), 374-375.

Buzzi, Stéphane, Jean-Claude Devinck et Paul-André Rosental (2006) La santé au travail: 18802006, Paris: La Découverte.

Caroly, Sandrine, Marie Bellemare, Philippe Davezies, Bernard Dugué, Fabien Coutarel, Alain Garrigou, Aurélie Landry, Johan Petit, Nadine Poussin et Daniel Prud'homme (2016) 
"Les conditions d'une activité collective entre les acteurs de la prévention des risques professionnels: enjeux de pluridisciplinarité », Rapport de recherche PACTE/ANSES, Pluriprev. EEST/2012/13.

Caroly, Sandrine (2013) «Les conditions pour mobiliser les acteurs de la prévention des TMS: construire du collectif de travail entre pairs pour développer le métier et favoriser le travail collectif pluriprofessionnel», PISTES, 15 (2), 25 pages. [En ligne] «https://journals.openedition. org/pistes/3400».

Caroly, Sandrine (2010) Activité collective et réélaboration des règles: des enjeux pour la santé au travail, thèse de doctorat, Université Victor Segalen Bordeaux 2, École doctorale Sciences sociales: société, santé, décision, Habilitation à diriger des recherches, 258 pages.

Caroly, Sandrine, Céline Cholez, Aurélie Landry, Philippe Davezies, Nadine Poussin, Marie Bellemare, Fabien Coutarel, Alain Garrigou, Karine Chassaing, Johan Petit, Geneviève BarilGingras, Daniel Prud'homme et Perrine Parrel (2011) «Les activités des médecins du travail dans la prévention des TMS: ressources et contraintes», Rapport ANR SEST, https://halshs. archives-ouvertes.fr/halshs-00676973.

Caroly Sandrine, Fabien Coutarel, Evelyne Escriva, Yves Roquelaure, Jean-Michel Schweitzer et François Daniellou, coord. (2008) La prévention durable des TMS: Quels freins ? Quels leviers d'action? Lyon: Agence nationale pour l'avancement des conditions de travail, Direction Générale du Travail, 180 pages. [En ligne] «https://halshs.archives-ouvertes.fr/ halshs-00373778/document».

Cholez, Céline, Sandrine Caroly, Aurélie Landry et Dominique Vinck (2011) «L'interdisciplinarité dans la santé au travail: dynamique de l'apprentissage pratique et croisé en situation », dans Frédéric Darbellay et Theres Paulsen, Au Miroir des Disciplines, Peter Lang: Bern, p. 35-56.

Coté, Julie, Susie Ngomo, Susan Stock, Karen Messing, Nicole Vézina, David Antle, Alain Delisle, Marie Bellemare, Marie Laberge et Marie St-Vincent (2013) «Ten Years of Research on Work-related Musculoskeletal Disorders in Quebec: Towards Better Understanding for Better Prevention », Relations industrielles/Industrial Relations, 68 (4), 643-660.

Clot, Yves (2006) La fonction psychologique du travail, Paris: Presses Universitaires de France.

D'amour, Danielle, Marcela Ferrada-Videla, Leticia San Martin Rodriguez et Marie-Dominique Beaulieu (2005) «The Conceptual Basis for Interprofessional Collaboration: Core Concepts and Theoretical Frameworks », Journal of Interprofessional Care, 19, Suppl. 1, 116-131.

Davezies, Philippe (2007) «Intensification. Danger: le travail rétréci», Santé et travail, (57), 30-33.

Davezies, Philippe (1998) «De la récupération psychique», Santé et travail, (25), 35-37.

Dejours, Christophe (1993) «Coopération et construction de l'identité en situation de travail», Futur antérieur, 2 (16), 41-52.

Dellacherie, Christian, Paul Frimat et Gilles Leclercq (2010) La santé au travail. Vision nouvelle et professions d'avenir, Rapport remis aux Ministre du Travail, de la Solidarité et de la Fonction publique, Ministre de l'Enseignement supérieur et de la Recherche, Ministre de la Santé et des Sports, Québec: Édition Gouvernement du Québec.

Drouin, Louis, Robert Simard, Gisèle Fontaine et Claude Huneault (2004) «L'élaboration et la mise en application des programmes de santé spécifiques aux établissements: I'approche québécoise », Santé publique, 16 (2), 329-337.

Ferré, Nathalie (2010) «Les institutions et les acteurs en santé au travail au Québec et en France: regard croisé», Perspectives interdisciplinaires sur le travail et la santé [En ligne], 12-1 I 
2010, mis en ligne le 01 février 2010, consulté le 22 septembre 2015. URL: https://journals. openedition.org/pistes/1618.

Fontaine, Gisèle, Cora Brahimi et Marie Fortier (2013) Référentiel de compétences en santé au travail du réseau de la santé publique du Québec, Québec: Institut nationale de santé publique du Québec, Direction des risques biologiques et de la santé au travail, 83 pages. [En ligne] « https:// www.inspq.qc.ca/sites/default/files/publications/1719_refecompsantetravsantepublqc.pdf ».

Huez, Dominique (1998) "Santé et précarisation du travail», dans Jacques Lebas et Pierre Chauvin (dir.), Précarité et santé, Paris: Flammarion, p. 143-154.

Marichalar, Pascal (2010) «La médecine du travail sans les médecins? », Politix, (3), 27-52.

Lecocq, Charlotte, Bruno Dupuis, Henri Forest et Hervé Lanouzière (2018) Santé au travail: vers un système simplifié pour une prévention renforcée, Rapport au Premier ministre, 174 pages. [En ligne] «https://www.ladocumentationfrancaise.fr/rapports-publics/184000578/index. shtml».

Organisation mondiale de la Santé (1999) Glossaire de la promotion de la santé, Genève: OMS. [En ligne] «https://apps.who.int/iris/bitstream/handle/10665/67245/WHO_HPR_HEP_98.1_ fre.pdf », consulté le 6 décembre 2015.

Plante, Robert, Luc Bherer et Michel Vézina (2010) «Comment protéger la santé des travailleurs dans un système qui ne repose pas sur les notions d'aptitude et d'inaptitude? », Perspectives interdisciplinaires sur le travail et la santé. [En ligne], PISTES, 12 (1), mis en ligne le 01 février 2010, consulté le 07 octobre 2015. URL: http://pistes.revues.org/1687.

Plante, Robert et Luc Bherer (2006) «La médecine du travail au Québec: une pratique de santé publique», Santé, société et solidarité, 2, 15-21.

Poussin, Nadine (2015) «Développement des sentiments au travail : quel développement du pouvoir d'agir? ", dans Actes du 50 Congrès de la Société d'Ergonomie de Langue Française, "Articulation performance et santé dans l'évolution des systèmes de production», 23-29 septembre, Paris, France, p. 510-515.

Prud'homme, Daniel (2019) La collaboration interprofessionnelle dans les équipes de santé au travail, thèse de doctorat, Département des relations industrielles, Université Laval, Québec, 301 pages.

Prud'homme, Daniel (2011) Les interventions en prévention des troubles musculosquelettiques par les équipes de santé au travail: une étude exploratoire, mémoire de maîtrise, Département des relations industrielles, Université Laval, Québec, 166 pages.

Prud'homme, Daniel, Marie Bellemare et Geneviève Baril-Gingras (2011) «Les interventions visant la prévention des TMS au Québec: la pratique des équipes de santé au travail », dans Caroly Sandrine (coord.) et al., Les activités des médecins du travail dans la prévention des TMS: ressources et contraintes, ANR-SEST09, 30 pages.

Salman, Scarlett (2008) «Fortune d'une catégorie: la souffrance au travail chez les médecins du travail », Sociologie du travail, 50 (1), 31-47.

Stock, Susan, France Tissot et Faïza Lazreg (2014) Évaluation de l'implantation du programme de prévention des TMS liés au travail du Réseau de santé publique en santé au travail. Synthèse, Québec: INSPQ, 21 pages. [En ligne] «https://www.inspq.qc.ca/pdf/publications/1832_Evaluation_Troubles_Musculo-Squelettiques.pdf », (page consultée le 3 mai 2016).

Stock, Susan, Diane Caron, Louis Gilbert, Lise Gosselin, Ghislaine Tougas et Alice Turcot (2006) La prévention des troubles musculosquelettiques reliés au travail; réflexion sur le rôle du 
Réseau de la santé publique et orientations proposées pour la santé au travail, Québec: INSPQ. [En ligne] https://www.inspq.qc.ca/sites/default/files/publications/428-preventiontrou blesmusculosquelettiques.pdf, (page consultée le 3 mai 2016).

Terssac, Gilbert De et Corinne Chabaud (1990) «Référentiel opératif commun et fiabilité», dans Jacques Leplat Et Gilbert De Terssac (dir.), Les facteurs humains de la fiabilité dans les systèmes complexes, Marseille: Octarès, p. 111-140.

Tougas, Ghislaine (2014) « Comment amener une entreprise à s'intéresser à la prévention des TMS? Une démarche de mobilisation basée sur les modèles de changement du comportement», Comptes-rendus du 45e Congrès de l'Association canadienne d'ergonomie, Montréal, 7 au 9 octobre 2014.

Vézina, Nicole (2010) "TMS ailleurs. Prévention des TMS au Québec», Archives des maladies professionnelles et de l'environnement, 71, 426-430.

\section{RÉSUMÉ}

\section{Travail collectif pluridisciplinaire dans la prévention} des risques professionnels complexes: ressources et contraintes au Québec et en France

Dans le cadre d'une étude menée au sein de services de santé au travail au Québec et en France, nous nous intéressons aux conditions favorables aux pratiques de travail collectif pluridisciplinaire (TCP). Face à des problèmes de santé complexes, tels que les troubles musculosquelettiques (TMS) ou les troubles de santé psychologique (TPS), des professionnels de différents métiers sont amenés à collaborer, notamment pour mener des actions de prévention primaire ayant pour cible les conditions de réalisation du travail. Le contexte dans lequel œuvrent, d'une part, les équipes de santé au travail (ESAT) au Québec et, d'autre part, les services de santé au travail interentreprises (SSTi) en France diffèrent en raison de leur histoire, leurs cadres juridique et institutionnel, leurs missions et dispositifs.

En étudiant les actions en milieu de travail menées par les acteurs de ces services, à partir de données recueillies par le biais d'observations et d'entretiens réalisés dans les deux contextes, nous examinons les formes de travail collectif pluridisciplinaire en prévention des TMS. Ceci nous a permis de mettre en lumière les contraintes et les ressources qu'offre, à cet égard, chacun des contextes. Nous constatons que le contexte québécois, avec son approche de santé publique qui prescrit une démarche structurée faisant appel à la pluridisciplinarité, permet des formes plus élaborées de travail collectif grâce au partage d'outils communs et aux prises de décisions collectives quant aux modalités de l'intervention. Dans le contexte français, bien que les formes du travail collectif pluridisciplinaire soient moins développées, le mandat de prévention assuré par les services de santé au travail permet un élargissement du périmètre d'action que l'on ne retrouve pas dans le contexte québécois. Ces résultats interrogent les marges de manœuvre offertes par les dispositifs en vue d'une prévention durable et efficace des risques professionnels.

MOTS-CLÉs: intervention, troubles musculosquelettiques, médecine du travail, santé au travail, Québec, France. 


\section{SUMMARY}

\section{Collective Multidisciplinary Work in the Prevention of Complex Occupational Risks: Resources and Constraints in Quebec and France}

Based on a study conducted in the Occupational Health Services sector in Quebec and France, we examine the conditions that are favourable to collective multidisciplinary work practices (TCP). In order to deal with complex issues like musculoskeletal disorders (MSD) or psychological health disorders (TPS), professionals from different disciplines are called upon to collaborate, and in particular to put in place, primary prevention actions focused on conditions related to the carrying out of work. The context in which these teams, called occupational health teams (ESAT) in Quebec and inter-company occupational health services (SSTi) in France, operate differs in terms of historical background, legal and institutional framework, mission, and operation.

By studying actions in the workplace carried out by the actors of these services and from data collected through observations and interviews in both contexts, we examine the forms of collective multidisciplinary work done relating to MSD prevention. This enables us to highlight the constraints and resources that each of these contexts offers in this respect. We find that the Quebec context, with its approach to public health that prescribes structured multidisciplinarity, allows for more advanced forms of collective work through the sharing of common tools and collective decision making in relation to methods of intervention. In the French context, although forms of collective multidisciplinary work are less developed, the prevention mandate of the occupational health services allows for a greater range of action that is not found in the Quebec context. These findings question the room for manoeuvre offered by these approaches for the sustainable and effective prevention of occupational risks.

KEYWORDS: intervention, musculoskeletal disorders, occupational health, Quebec, France.

\section{RESUMEN}

\section{Trabajo colectivo multidisciplinario en la prevención de riesgos ocupacionales complejos: recursos y limitaciones en Quebec y Francia}

En el marco de un estudio en el seno de los servicios de salud ocupacional en la provincia de Quebec y en Francia, nos interesamos en las condiciones favorables a las prácticas de trabajo colectivo pluridisciplinario (TCP). Ante los problemas de salud complejos, como los trastornos musculo-esqueléticos (TME) o los trastornos psicológicos (TP), los profesionales de diferentes profesiones deben colaborar, especialmente para llevar a cabo acciones de prevención primaria centradas en las 
condiciones de realización del trabajo. El contexto en el cual trabajan, de un lado, los equipos de salud ocupacional (ESAT) en Quebec y, de otro lado, los servicios de salud ocupacional inter-empresas (SSTi) en Francia, difieren en cuanto a su historia, sus marcos jurídico e institucional, sus misiones y dispositivos.

Al estudiar las acciones en el medio de trabajo llevadas a cabo por los actores de dichos servicios y a partir de datos recolectados por medio de observaciones y entrevistas realizadas en dos los contextos, examinamos las formas de trabajo colectivo pluridisciplinario en prevención de los TME. Esto nos ha permitido resaltar las limitaciones y los recursos que ofrece cada contexto al respecto. Constatamos que el contexto quebequense con su enfoque de salud pública que prescribe un procedimiento estructurado incitando a la multidisciplinariedad, permite formas más elaboradas de trabajo colectivo gracias al hecho de compartir instrumentos comunes y a la toma de decisiones colectivas con respecto a las modalidades de la intervención. En el contexto francés, aunque las formas de trabajo colectivo multidisciplinario sean menos desarrolladas, el mandato de prevención proporcionado por los servicios de salud ocupacional permite ampliar el alcance de la acción, lo que no se encuentra en el contexto quebequense. Estos resultados cuestionan los márgenes de maniobra ofrecidos por los dispositivos para una prevención durable y eficaz de los riesgos profesionales.

PALABRAS CLAVES: intervención, trastornos musculo-esqueléticos, medicina del trabajo, salud ocupacional, prevención, Quebec, Francia. 